Copyright C1997, American Institute of Aeronautics and Astronautics, Inc.

AIAA Meeting Papers on Disc, January 1997

A9715633, F49620-94-1-0133, NAGW-1356, NGT-10034, AIAA Paper 97-0598

\title{
Molecular dynamics studies of transport properties of supercritical fluids
}

\author{
Obika C. Nwobi
}

Pennsylvania State Univ. University Park

Lyle N. Long

Pennsylvania State Univ. University Park

Michael M. Micci

Pennsylvania State Univ. University Park

\begin{abstract}
AIAA, Aerospace Sciences Meeting \& Exhibit, 35th, Reno, NV, Jan. 6-9, 1997
Current rocket motors, gas turbines, and many projected advanced combustor designs operate supercritically or near supercritical conditions. While there have been efforts to use computational fluid dynamics to predict these flows, it is extremely difficult to obtain the necessary data on the transport properties under these conditions. In this paper, the determination of transport coefficients of supercritical fluids using a molecular dynamics method and the Green-Kubo formulas is described. Preliminary results for bulk liquid argon, nitrogen, and oxygen are compared with experimental and NIST values. Results are presented for subcritical, near-critical, and supercritical conditions. The computer code is written in FORTRAN-77 and uses the message passing interface (MPI). Most of the computer runs are made on an IBM SP2 computer. The performance of the code on the SP2 is compared to benchmark timing on various parallel architectures. (Author)
\end{abstract}




\title{
Molecular Dynamics Studies of Transport Properties of Supercritical Fluids
}

\author{
Obika C. Nwobi; Lyle N. Long; and Michael M. Micci ${ }^{\ddagger}$ \\ Department of Aerospace Engineering \\ University Park, PA 16802
}

\begin{abstract}
Current rocket motors, gas turbines, and many projected advanced combustor designs operate supercritically or near supercritical conditions. While there have been efforts to use computational fluid dynamics (CFD) to predict these flows, it is extremely difficult to obtain the necessary data on the transport properties under these conditions. In this paper, the determination of transport coefficients of supercritical fluids using a molecular dynamics method and the Green-Kubo formulae is described. Preliminary results for bulk liquid argon, nitrogen, and oxygen are compared with experimental and NIST values. Results are presented for subcritical, near critical, and supercritical conditions. The computer code is written in Fortran-77 and uses the message passing interface (MPI). Most of the computer runs are made on an IBM SP2 computer. The performance of the code on the SP2 is compared to benchmark timing on various parallel architectures.
\end{abstract}

\section{Introduction}

Current rocket motors, gas turbines, and many projected advanced combustor designs operate supercritically or near critical conditions. ${ }^{1}$ Computational fluid Dynamics (CFD) has been used to model Droplet evaporation and combustion in supercritical and near critical environments by several researchers including Yang et al., ${ }^{2}$ Matlosz et al., ${ }^{3}$ Rosner and Chang, ${ }^{4}$ Kadata and Hiroyasu, ${ }^{5}$ Jin and Borman, ${ }^{6}$ Curtis and Farrel, ${ }^{7}$ and Hsieh et al. ${ }^{8}$ Quantitative information on the coefficients of mass, momentum and energy transport, and the equation of state are very important in these studies. However, in many complex systems such as rarefied flows, supercritical environments, shock regions, and highly-energized

\footnotetext{
${ }^{*}$ Research Assistant

${ }^{\dagger}$ Associate Professor, Senior Member, AIAA

$¥$ Associate Professor, Senior Member, AIAA

${ }^{\circ}$ (C) 1997 by Nwobi, Long and Micci. Published by the American Institute of Aeronautics and Astronautics, Inc. with permission.
}

plasmas, these coefficients are not known accurately. As a result the necessary information is lacking in the use of the CFD methods for simulating supercritical evaporation and combustion.

Although the transport properties of some fluids have been calculated via molecular dynamics (MD), ${ }^{9}$ only limited success have been achieved for molecular fluids which are essential for the supercritical droplet studies. The transport coefficients of atomic fluids and fluid mixtures such as argon, krypton etc. have been correctly computed and the results compare well with experiments. However for molecular fluids, the properties have not been obtained to acceptable accuracy levels. ${ }^{10}$ This is mainly due to the inadequate modeling of the long-time tail behaviors of the relevant autocorrelation functions, or to the use of incorrect potential model parameters. An attempt is made herein to compute the transport properties of bulk fluids using the Green-Kubo formulae. Initial studies were focused on liquid argon and this has been extended to oxygen and nitrogen at various operating conditions.

\section{Molecular Dynamics}

In theory the governing equation for liquids is the $6 \mathrm{~N}$ dimensional Liouville equation. ${ }^{11}$ However, it is not feasible to solve the Liouville equation and it is of limited direct use. The Boltzmann equation can be derived from the Liouville equation if one assumes a dilute gas. If one also assumes that the gradients are small (or the Knudsen number is small), then one can derive the NavierStokes equations from the Boltzmann equation. However, the Navier-Stokes and Boltzmann equations are not strictly valid for liquids. While the basic conservation laws hold for liquids, the transport properties and equations of state are very difficult to derive. An alternative to this approach is to use direct simulation.

Molecular dynamics (MD) and Monte Carlo (MC), a stochastic method, are very widely used direct simulation techniques. The advantage of these techniques for the solution of complex reacting flow problems is that all attendant physical phenomena: viscosity, surface ten- 
sion, evaporation, and reaction rates; come from the force potential between the particles. These methods are especially useful for simulating dilute systems, and as the capability of computers increases, they will be more useful at higher number densities. Direct simulation methods do not require the use of empirical submodels for each physical process or temperature dependent physical properties.

$\mathrm{MD}$, the technique used in the present study, involves the solution of the equations of motion of a system of molecules that interact with each other through an intermolecular potential. ${ }^{12}$ Provided that an accurate potential can be found for the system of interest, MD can be used regardless of the phase and thermodynamic conditions of the substances involved. Although computationally intensive, this method requires no a priori assumptions regarding geometrical symmetry, transport properties, or thermodynamic behavior. All calculations are performed from first principles based on intermolecular potentials, and thermodynamic and transport properties are results instead of assumptions.

The MC method, like MD aims to generate a trajectory in phase space which samples from a chosen statistical ensemble. However, unlike MD, it is used mainly for systems in equilibrium. ${ }^{12}$ The number of $\mathrm{MC}$ simulations required for evaluating simple thermodynamic properties of systems such as temperature and the virial is normally higher than the corresponding MD timesteps. ${ }^{13}$ Hence, the technique is not suitable for the determination of transport coefficients.

\subsection{Potentials}

Several mathematical representations of the intermolecular force have been used including the rigid impenetrable sphere, the square-well potential, and the point center of repulsion potential. ${ }^{14}$ The most commonly used potential is the Lennard-Jones (LJ) $12-6$ potential $^{12}$

$$
\Phi^{L J}(r)=4 \varepsilon\left((\sigma / r)^{12}-(\sigma / r)^{6}\right)
$$

where $\sigma$ is the zero energy separation distance, $\epsilon$ is the minimum energy and $r$ is the radial distance. The sixthpower term is attractive out to long ranges and can be derived from quantum mechanics where it is referred to as the induced-dipole-induced-dipole interaction. The twelfth-power term is repulsive and acts primarily at short ranges. There is no rigorous derivation of the value of twelve other than that it appears to accurately model the repulsive force when compared to experiments and has been accepted as a standard world wide to facilitate communication and comparison of results. The repulsive term is sometimes replaced with an exponential, however the solutions are relatively insensitive to the exact form used for the repulsive term. Since the assumed potential seeks to represent all the forces acting on an atom or molecule, there is no need for additional models and the above potentials are valid with constant values of $\sigma$ and $\epsilon$ as one moves from solid to the liquid to the gas phase, however, minor corrections at very high pressures have been suggested. ${ }^{14}$ Pairwise potentials are, therefore, very commonly used in $\mathrm{MD}$ and they reduce the number of computations when compared to many-body potentials.

However, for certain systems the pairwise potentials do not accurately model known behaviors. The force between particles, in these cases, depend on the position of all other particles in the vicinity. Thus in addition to the force between pairs of particle, a force that acts on triplets of particles is introduced ${ }^{13}$ For example silicon and gallium arsenide crystallize in a diamond lattice with each atom having four neighbors in a tetrahedral arrangement. Hence, they are better modeled by threebody potential. The Tersoff potential is very commonly used for modeling silicon. ${ }^{15}$ It is given by

$$
\Phi_{T}=\sum_{i} \sum_{j}\left[\Phi_{R}\left(r_{i j}\right)+b_{i j} \Phi_{A}\left(r_{i j}\right)\right]
$$

The $\Phi_{A}$ term indicates the attractive part, while the $\Phi_{R}$ is the repulsive part, and

$$
b_{i j}=\left(1+\beta^{n} \zeta_{i j}^{n}\right)^{-n / 2}
$$

with

$$
\begin{gathered}
\zeta_{i j}=\sum_{k \neq i, j}^{N} g\left(\theta_{i j k}\right) \exp \left[\lambda_{3}^{3}\left(r_{i j}-r_{i k}\right)^{3}\right] \\
g\left(\theta_{i j k}\right)=1+\frac{c^{2}}{d^{2}}-\frac{c^{2}}{\left[d^{2}+\left(h-\cos \theta_{j i k}\right)^{2}\right]}
\end{gathered}
$$

where $\beta, \zeta, \lambda_{3}, c, d$, and $h$ are parameters. There are cut-off functions in the Tersoff potential which allow for efficient computations.

The intermolecular force is obtained from the spatial gradient of the potential. For the Lennard-Jones potential this is given by

$$
\mathbf{f}_{i j}=-\frac{24 \varepsilon}{r^{2}}\left(2(\sigma / r)^{12}-(\sigma / r)^{6}\right) \mathbf{r}_{i j}
$$

where $\mathbf{r}_{i j}$ is the the position vector between two particles $i$ and $j$. Given the total force on a specified particle and using Newton's second law of motion, the acceleration of the particle can be determined from $a=f / m$. Using finite differences and the knowledge of this acceleration, the original velocity, and position, the particle can be moved to its new position and assigned a new velocity at a certain time in the future. Repeating this step for every particle in the system and recomputing the forces on each of the particles, results in the completion of one time step. The process continues over a given number 
of time steps. The determination of the forces requires more than $75 \%$ of the total computation time. This is due to the fact that the pair interactions between the particles have to be calculated and summed up. This often requires a double "Do-Loop". Hence, this part of the computer program has to be carefully optimized to reduce execution time. ${ }^{9}$

\subsection{Modeling of Diatomics}

While the above discussion is strictly valid for any system of atoms, to simulate molecular fluids, additional measures have to be taken. Molecules can be modeled by one of two means: treating each atom in the molecule as a separate Lennard-Jones site (site-site); or by defining a central site with corrections for angular dependency. Luo and Hoheisel ${ }^{16}$ modeled nitrogen using both methods and found that they gave the same results. The central site method starts with the Newton-Euler equations of motion. Because singularities appear when written in terms of Euler angles, the equations are written in terms of quaternions which are then solved in matrix form. ${ }^{12}$ The numerical algorithm for solving the Euler equations is very complicated and, hence, this method is not widely used.

The site-site method consists of solving the ordinary equations of motion for each atomic site subject to a constraint which is the fixed bond length. ${ }^{12,13}$ This is given by the following equation:

$$
\gamma_{B}=\left|\mathbf{r}_{\alpha}^{i}-\mathbf{r}_{\alpha}^{j}\right|^{2}-d_{i j}^{2}=0
$$

where $\gamma_{B}$ is the constraint and $d_{i j}^{2}$ denotes the desired separation distance between atoms $i$ and $j$ of a given molecule $\alpha . \quad \mathbf{r}_{\alpha}^{i}$ and $\mathbf{r}_{\alpha}^{j}$ are the radial positions of the two atoms. Usually the bond constraint suffices to keep the structure of the molecule for the dynamics, and it results in a force which is added to the potential force. The molecular interaction generally consists of pairwise additive atom-atom interactions. The potential between two molecules $\alpha$ and $\beta$ is given by

$$
v_{\alpha \beta}=\sum_{i=1}^{n} \sum_{j=1}^{n} \Phi\left(\mathbf{r}_{\alpha \beta}^{i j}\right)
$$

where $\Phi$ is the center-center potential function, while $\mathbf{r}_{\alpha \beta}^{i j}$ indicates the position vector between two atomic centers $i, j . n$ is the number of atoms per molecule.

Constraint dynamics seems to be the method of choice for diatomics and it is used in this study. The technique involves writing the equations of motion to include the constraint force due to the fixing of the bond length. The magnitude of the constraint forces are determined iteratively using undetermined multipliers and the velocity Verlet finite difference scheme. Typically, the iterative process requires about $5-10 \%$ of the total computation time. To solve the equations of motion under constraints, firstly, the positions and the velocities of all the atoms in the system are determined without the constraints. Then, these values are corrected with the incorporation of the constraints.

\subsection{Radial Distribution Function}

It is customary in MD simulations to also examine static properties of the molecules. An indication of whether a given substance is a solid, liquid, or gas is obtained by examining the pair (or radial) distribution function, $g(r)$. This function gives the probability of finding a pair of molecules a distance $r$ apart, relative to the probability expected for a completely random distribution at the same density. Since the function depends on both density and temperature of a given substance, its shape is distinctly different for the three phases.

The radial distribution function can be determined experimentally by evaluating the diffraction of $\mathrm{X}$-rays, neutrons or electrons. The function can also be readily computed during an MD simulation by using the system trajectories. The Lennard-Jones parameters used for simulating oxygen were obtained with the help of the radial distribution function. Using the most common L$\mathrm{J}$ parameters for oxygen as a starting point, the radial function was obtained and compared to the experimentally determined function as shown in figure $1 .^{17}$ The L-J parameters were systematically varied until the closest match was obtained between the two $g(r)$ functions.

\section{Computation of Transport Co- efficients}

\subsection{Correlation Functions}

In computer simulations, transport coefficients can be calculated from equilibrium correlation functions, by observing Einstein relations, or by conducting a suitable non-equilibrium MD simulation. ${ }^{12}$ In the present study the transport coefficients were computed using GreenKubo formulae in which a correlation function is integrated over time. ${ }^{10,12,18}$ The autocorrelation functions (ACF) provide a powerful tool for describing the dynamics of liquids and they give insight into the microscopic behavior of fluids. An ACF can be derived from the Enskog kinetic equation and the corresponding transport coefficient obtained via the linear response theory with the use of hydrodynamic equations. ${ }^{10}$ The ACF of a real dynamic variable $A(t)$ measures the relation between the values of the variable at two different times. It is given by the following expression: 


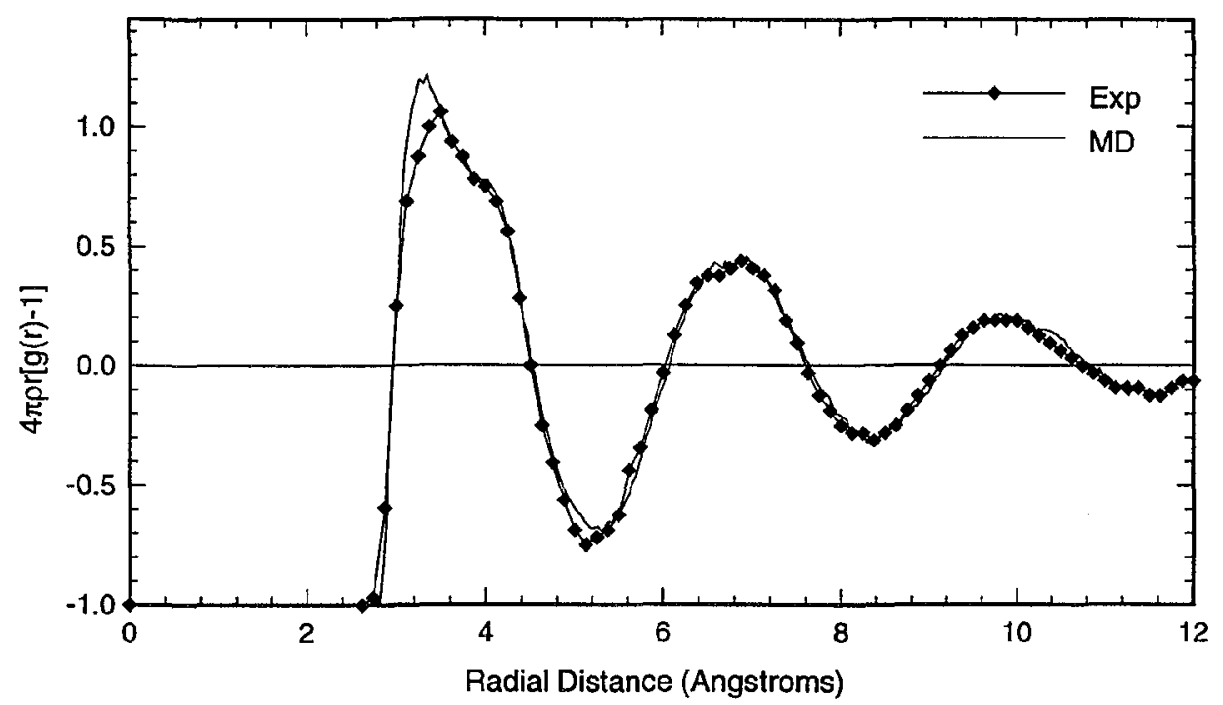

Figure 1: Comparison of MD and Experimental Radial Distribution Functions for Oxygen at $84 \mathrm{~K}$ and $1.167 \mathrm{~g} / \mathrm{m}^{3}[17]$.

$$
C(t)=\lim _{\tau \rightarrow \infty} \frac{1}{\tau} \int_{0}^{\tau} A\left(t_{0}\right) A\left(t_{0}+t\right) d t_{0}
$$

This can be written in the form

$$
C(t)=\left\langle A\left(t_{0}\right) A\left(t_{0}+t\right)\right\rangle
$$

where $t_{0}$ and $\mathrm{t}$ denote an arbitrary time and the time difference, respectively, and (...) denotes a time average. ACFs can be classified into either one-particle or collective functions. The former refers to the time-dependent motion of single particles of the system, while the latter refers to the time-dependent behavior of all the particles of the system. The collective ACFs are usually less precisely computed than the single ACFs during MD simulations because statistical precision cannot be improved by summing over the ACF of all the particles of the system. The integral of an ACF yields the corresponding transport coefficient. The integral over the velocity ACF yields the self-diffusion coefficient, while the thermal conductivity is represented by the integral over the heat current ACF. The off-diagonal elements of the stress tensor give the shear viscosity coefficient, while the bulk viscosity is given by the diagonal terms.

Figure 2 presents normalized ACFs as functions of delay time. For most conditions, the ACFs decay nearly to zero within about $1.0 \mathrm{ps}$ (about 5,000 timesteps if a step size of 2 femtoseconds is used). However, whereas the ACFs for shear viscosity and thermal conductivity have only positive values throughout the time delay, the diffusion ACF often shows a significant negative part. This negative part is due to the fact that the dynamic variable for self-diffusion is composed of only the kinetic part and at certain times the particle velocity may be reversed due to collisions with other particles. The motion of this particle is then retarded by the backflow created by the other particles. ${ }^{19}$ The negative part increases with the density of the given substance. The Green-Kubo integrands consist of a steep short time branch and a subsequent long time branch of up to $2 \mathrm{ps.} \mathrm{Hence,} \mathrm{to}$ accurately compute the transport coefficients, the long time tails must be accounted for. From mode-coupling theory, it has been predicted that the diffusion and the shear viscosity ACFs have long time tails of the following forms ${ }^{20}$

$$
\begin{gathered}
f_{D}(t)=\alpha_{D} t^{-3 / 2} \\
f_{\mu}(t)=\alpha_{\mu} t^{-3 / 2}
\end{gathered}
$$

$\alpha_{D}$ and $\alpha_{\mu}$ are constants and they depend on the fluid properties. In the present study, the tails were fitted to 
polynomials of the form $f(t)=A t^{-3 / 2}$.

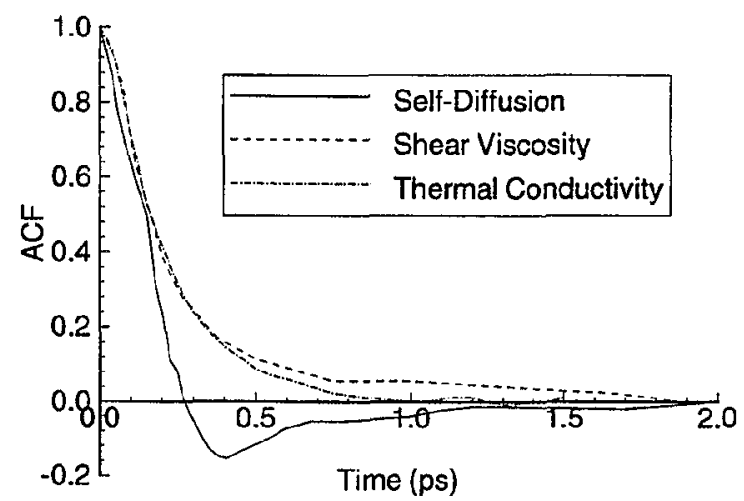

Figure 2: Normalized ACF Plots for Argon at the Triple Point [9].

The numerical accuracy of ACFs for molecular fluids computed by MD are influenced by such factors as the system size, length of simulation, number of time origins, and the cut-off radius of the potential function. Hoheise ${ }^{10}$ found that a truncation of $2.5 \sigma$ radius for the LJ potential and system size of 108 molecules is sufficient to generate reliable ACFs for the transport coefficients at points away from the critical point. Closer to the critical point larger sizes of at twice least the correlation length have to be used. ${ }^{21}$ For most conditions, simulations of about half a million time steps suffice to give accurate ACFs. The ACFs can also be adversely affected by the periodic boundary conditions which are used in MD simulations. Unrealistic correlations can be obtained when the delay time exceeds the periodic correlation time. ${ }^{10}$

\subsection{Green-Kubo Formulae}

The use of the Green-Kubo formulae for the determination of transport coefficients depends on the evaluation of a microscopic current at a given time $t$ subsequent to an initial time $t_{0}$ by the application of an MD simulation. This generates an $N$-particle trajectory with the ensemble average replaced by an average over "time origins" that are equally spaced (in time) along the trajectory.

The diffusion coefficient relates particle flux to a concentration gradient. The self-diffusion process is the mass transport associated with the motion of a single particle in a fluid and, hence, characterizes a one-particle property of the system. Self-diffusion may be computed using the center of mass velocity of a marked particle of the fluid. ${ }^{9}$ The Green-Kubo formula contains the velocity autocorrelation function and it is given by

$$
D=\frac{1}{3 N} \int_{0}^{\infty} \sum_{\alpha=1}^{N}\left\langle\mathbf{v}_{\alpha}\left(t_{0}\right) \cdot \mathbf{v}_{\alpha}\left(t_{0}+t\right)\right\rangle d t
$$

where $D$ denotes the mean self-diffusion of the system. This mean value is obtained by summing the velocity autocorrelation function of each of the particles over the total number $N$. The ACF is composed of only kinetic terms and, hence, takes the least computation time of all the three correlation functions being considered in the present study since it does not depend on intermolecular forces.

The shear viscosity coefficient measures the resistance of the fluid to a shearing force, and it gives an indication of the shear stress induced by an applied velocity. The correlation function contains the non-diagonal elements of the stress tensor and it is a collective property of the entire fluid. ${ }^{10}$ The Green-Kubo formula is given by

$$
\mu_{x y}=\frac{1}{V k_{B} T} \int_{0}^{\infty}\left\langle J^{x y}\left(t_{0}\right) J^{x y}\left(t_{0}+t\right)\right\rangle d t
$$

where $J^{x y}$ denotes a non-diagonal term of the stress tensor and it is given by

$$
f^{x y}=m \sum_{\alpha=1}^{N} v_{\alpha}^{x} v_{\alpha}^{y}-\frac{1}{2} \sum_{\alpha=1}^{N} \sum_{\beta=1}^{N} \sum_{i=1}^{n} \sum_{j=1}^{n} R_{\alpha \beta}^{x} \frac{\partial \Phi\left(\mathrm{r}_{\alpha \beta}^{i j}\right)}{\partial y}
$$

Here $T$ and $V$ denote the temperature and volume of the system, respectively, and $m$ denotes the mass of each particle. $\mathbf{R}_{\alpha \beta}$ denotes the difference vector between the center of mass position vectors of the molecules, while $\mathbf{r}_{\alpha \beta}^{i j}$ indicates the position vector between two atomic centers $i, j . \Phi$ denotes the pair interaction potential of different molecules, while $n$ and $k_{B}$ are the number of atoms per molecule and the Boltzmann constant, respectively. The shear stress ACF is composed of a kinetic term, which measures the correlation of momentum transport caused by atomic motions; a potential term which measures the correlation of momentum transport caused by interatomic forces, and a cross term, which measures the coupling of atomic motions and forces. Generally, the kinetic term dominates the ACF at low densities (gases), while the potential term dominates at higher densities (liquids). Statistical precision is improved by averaging over all the three terms $\left(\mu_{x y}, \mu_{x z}, \mu_{y z}\right)$ that result from the stress tensor.

The thermal conductivity coefficient measures the transport of heat in a system. The correlation function is obtained from the heat current and it is also a collective property of the entire fluid. ${ }^{10}$ The Green-Kubo formula is given by 


$$
\lambda_{x}=\frac{1}{V k_{B} T^{2}} \int_{0}^{\infty}\left\langle J^{x}\left(t_{0}\right) J^{x}\left(t_{0}+t\right)\right\rangle d t
$$

where $J^{x}$ denotes an arbitrary component of the heat current and it is given by

$$
\begin{gathered}
J^{x}=\frac{m}{2} \sum_{\alpha=1}^{N}\left|\mathbf{v}_{\alpha}\right|^{2} v_{\alpha}^{x} \\
-\frac{1}{2} \sum_{\alpha=1}^{N} \sum_{i=1}^{n} v_{\alpha}^{x} \sum_{\beta=1}^{N} \sum_{j=1}^{n}\left[\left(R_{\alpha \beta}^{x} \frac{\partial \Phi\left(\mathbf{r}_{\alpha \beta}^{i j}\right)}{\partial x}-\Phi\left(\mathbf{r}_{\alpha \beta}^{i j}\right) \mathbf{i}\right)\right]
\end{gathered}
$$

where $\mathrm{i}$ denotes a unit tensor. The ACF is also composed of kinetic, potential, and cross (kinetic-potential) terms. The computation of thermal conductivity takes the most time of the three transport coefficients that are being determined in the present study. Again, statistical precision is improved by averaging over all three components $\left(\lambda_{x}, \lambda_{y}, \lambda_{z}\right)$.

The corresponding formulae of the tensors for atomic fluids are

$$
\begin{aligned}
& J^{x y}=m \sum_{i=1}^{N} v_{i}^{x} v_{i}^{y}-\frac{1}{2} \sum_{i=1}^{N} \sum_{j=1}^{N} \mathbf{r}_{i j}^{x} \frac{\partial \Phi\left(\mathbf{r}_{i j}\right)}{\partial y} \\
& J^{x}=\frac{m}{2} \sum_{i=1}^{N}\left|\mathbf{v}_{i}\right|^{2} v_{i}^{x} \\
&-\frac{1}{2} \sum_{i=1}^{N} \sum_{j=1}^{N}\left[\left(r_{i j}^{x} \frac{\partial \Phi\left(\mathbf{r}_{i j}\right)}{\partial x}-\Phi\left(\mathbf{r}_{i j}\right) \mathbf{i}\right)\right] v_{i}^{x}
\end{aligned}
$$

where $\mathbf{r}_{i j}$ and $\Phi\left(r_{i j}\right)$ denote the position vector and the potentials, respectively, between atoms $i$ and $j$. Typically, the determination of the ACFs accounts for about $20 \%$ of the total computation time.

\section{Algorithm and Code Perfor- mance}

The MD computer code is written in Fortran-77 and it uses the message passing interface (MPI). Most of the runs were made on an IBM SP2 computer. The code uses a leap-frog Verlet integration algorithm, periodic boundary conditions and the Lennard-Jones 12-6 potential for the pair interactions. ${ }^{12,13,19}$ For modeling diatomics such as oxygen and nitrogen, each molecule is treated as a 2-site Lennard-Jones particle and "constraint dynamics" is used to maintain a constant bond length. Each simulation is done in a conventional $N E V \mathbf{p}$ ensemble, where $N$ denotes the particle number, $E$ the total energy, $V$ the volume and $\mathbf{p}$ the momentum.

\subsection{Finite Difference Method}

Using Newton's Second Law applied to each particle, the classical equations of motion of a system of $\mathrm{N}$ particles can be expressed in the form

$$
m_{i} \mathbf{a}_{i}=\mathbf{f}_{i}
$$

where $\mathbf{a}_{i}$ denotes the acceleration of a given particle. This generates $3 \mathrm{~N}$ second-order differential equations in the radial position of the atoms, $\mathbf{r}_{i}$. Given the total force on each particle, the acceleration can be determined and then the velocity obtained. Using these velocities and accelerations, the particle is moved a finite amount on the time step. An appropriate finite difference scheme must be simple, stable, accurate, and permit long time step size. The most widely used algorithm is the partially implicit velocity Verlet algorithm. However, this scheme handles velocities awkwardly, and it often introduces some numerical imprecision. ${ }^{12}$ The scheme used for this work is a modified version of the Verlet algorithm called the "Leap-Frog" scheme.

The algorithm is shown below:

$$
\begin{gathered}
\mathbf{v}\left(t+\frac{1}{2} \delta t\right)=\mathbf{v}\left(t-\frac{1}{2} \delta t\right)+\delta t \mathbf{a}(t) \\
\mathbf{r}(t+\delta t)=\mathbf{r}(t)+\delta t \mathbf{v}\left(t+\frac{1}{2} \delta t\right) \\
\mathbf{v}(t)=\frac{1}{2}\left(\mathbf{v}\left(t+\frac{1}{2} \delta t\right)+\mathbf{v}\left(t-\frac{1}{2} \delta t\right)\right)
\end{gathered}
$$

For this scheme the position of each particle is determined at integral time steps, while the velocity is determined at half time steps. The stored quantities are the position $\mathbf{r}(t)$ and the acceleration $\mathbf{a}(t)$ at the current time step together with $\mathbf{v}\left(t-\frac{1}{2}\right)$. This scheme also makes it very easy to adjust the simulation energy, which is achieved by the scaling of velocities.

\subsection{Periodic Boundary Conditions}

Because of the computationally intense nature of $\mathrm{MD}$ simulations, the system sizes tend to be relatively small. A major obstacle to such simulations is the large fraction of molecules which lie on the surface boundaries. Whether or not the cube is surrounded by a containing wall, molecules on the surface experience quite different forces from molecules in the bulk. The problem of surface effects can be overcome by implementing periodic boundary conditions.

Periodic boundaries refer to the method of assuming the central simulation cell to be replicated throughout space to form an infinite lattice. The image cells are each the same size and shape as the primary cell, and each of them contain $N$ particles which are images of the particles in the primary cell. The periodicity extends to 
the position and velocities of the images in the image cell. ${ }^{19}$ In the course of the the simulation, as a molecule moves in the original cell, its periodic image in each of the neighboring cells moves in exactly the same way. As a molecule leaves the central simulation geometry, an image of the molecule will enter the central geometry through the opposite face. During the simulation, only the position and the velocities of the particles in the primary cell are stored. The positions of the images can be computed, when needed, by suitable coordinate transformations. The potential energy, $\Phi(r)$, of the system contains contributions from various images of the primary cell.

Hence, an infinitely replicated system throughout space can be simulated using a system with periodic boundaries. So long as the range of the intermolecular potential is small, for example the effective LennardJones potential used in the study, the properties of this small, infinitely periodic system, and the macroscopic system which it represents, are the same. ${ }^{12}$ However, periodic boundary conditions can adversely affect the ACFs of the transport coefficients computed during MD simulations. Unrealistic correlation can be obtained when the delay time exceeds the periodic correlation time. ${ }^{10}$

\subsection{Performance Enhancements}

The one great limitation of $\mathrm{MD}$ is the computational demand. Hence, many techniques have been used to increase the speed and efficiency of the code. Firstly, a "minimum image" convention is used to reduce the number of force calculations, due to the incorporation of periodic boundary conditions. Since intermolecular forces decay rapidly beyond just a few atomic diameters, the effect of such forces may be neglected for any molecules outside the immediate vicinity of a molecule. Using this convention, the number of force calculations is reduced from infinity to $\frac{1}{2} N(N-1)$. Secondly, a cell index method is used for checking which molecules need to be included in the force calculation of each molecule. This is achieved by dividing the simulation box into small cells into which the molecules are sorted. Only molecules in the surrounding need to be considered for force calculations. Thirdly, a "linked list" method is used for fast sorting of the molecules into cells. ${ }^{12}$

The "linked list" refers to the linking of a list array with the head array during the neighbor search ${ }^{12}$ and the routine is used at every time step. The head array contains the largest molecule number within every cell, while the list has the rest of the sorted molecule numbers in descending order. If there are no molecules left in a cell, the list value will be zero. This method hence requires only the additional storage of two relatively small array pairs and its efficient use of memory is applied to track the neighbor locations.

\subsection{Parallel Aspects}

The method of parallelization currently being used is the particle decomposition method. This technique is implemented by distributing the particles evenly among the different processors. Each processor calculates the forces and displacements for the particles for which it is responsible. Then the new positions and velocities from each processor are broadcast to all other processors with which the next time step begins. Using the particle decomposition method, almost perfect load balancing has been achieved. ${ }^{22}$

The performance of the code on a 32 processor IBM SP2 has been compared to a benchmark timing of a bulk liquid simulation on various parallel architectures, ${ }^{23} .^{22}$ This is shown in figure 3 . The linear scaling with problem size evidenced by the benchmark runs is matched by the SP2 case. The simulation rate is consistently double the rate of the 512 processor nCUBE 2 and nearly four times faster than the 32 processor Intel iPSC/860. Only the 512 processor Intel Paragon and the 256 processor Cray T3D show better performance because of the higher peak speeds of the computers.

We also have another code that is used for small numbers of molecules. This code also uses MPI which essentially allows us to run many copies of a serial code simultaneously for different input conditions. This code by definition has $100 \%$ parallel efficiency. This is the code used for most of the results in this paper since the computation of the transport properties require relatively small systems of less than 1000 molecules.

\section{Molecular Dynamics Simula- tions}

Simulations have been performed for bulk liquid argon, nitrogen, and oxygen at various subcritical, supercritical and near critical conditions. The number of molecules used in the simulation, the time step size and the length of the runs were chosen to allow for the accurate evaluation of the Green-Kubo time correlation functions. The codes are currently being run on an IBM SP2 computer and each simulation typically takes about 24 hours. Useful details of the molecular dynamics simulations are shown in Table 1.

The MD simulations usually include initialization, equilibration, and time-dependent data acquisition. To start the simulation the molecules are initialized in a. face-centered cubic (FCC) lattice structure, and a Gaussian distribution is applied to initialize the velocities, with magnitudes conforming to the required temperature. The velocities are corrected such that the entire 


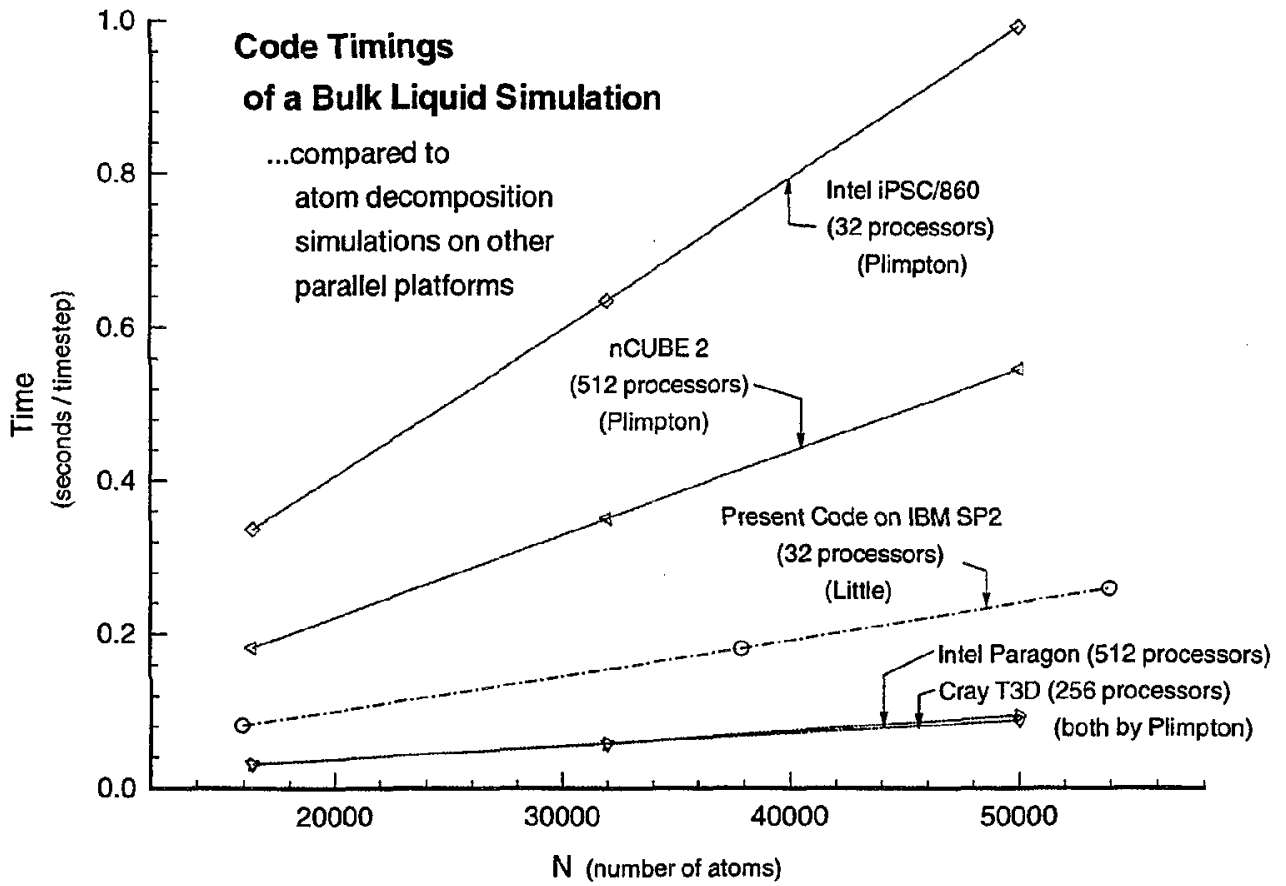

Figure 3: Parallel Code Performance Comparison [22][23].

Table 1: Technical Details of the MD Runs

\begin{tabular}{||c|c||}
\hline No. of Molecules & 256 \\
\hline Initial configuration lattice & FCC \\
\hline No. of equilibration time steps & 10,000 \\
\hline Total no. of time steps & 500,000 \\
\hline No. of averaging events for ACF & 10,000 \\
\hline Time step & $2 \times 10^{-15} s$ \\
\hline Integration Algorithm & Leap-frog Verlet \\
\hline Potential cut-off radius & $2.5 \sigma$ \\
\hline
\end{tabular}

system has no net momentum. The system is first allowed to reach an equilibrated state before the production run begins. The correlation functions are computed during the simulation. The velocity autocorrelation is computed outside the force subroutine, while the components of the other autocorrelation functions are calculated within it. Once the functions have been computed, Simpson's $1 / 3$ numerical integration rule is used to obtain the transport coefficients. Figure 4 shows a schematic of a typical MD simulation program. The sorting of molecules, calculation of forces, calculation and storing of ACF variables, and the integration of equations of motion are done at every time step.

\section{Results}

As a reference, the critical temperatures and pressures, and the Lennard-Jones parameters of the relevant elements are given in Table 2. Each simulation was performed for 500,000 time steps using a step size of $2 \times 10^{-15}$ seconds; with system sizes of 256 atoms for argon, and 256 molecules for nitrogen and oxygen. Results of the simulations performed on argon and nitrogen are presented in Table 3 and Table 4, respectively. The calculated shear viscosities and thermal conductivities are compared to values obtained from NIST software which uses equations to determine the transport coefficients of substances. ${ }^{24}$ The parameters of these equations were determined by experiments. ${ }^{25,26}$ Using experimental data, and with the help of the Benedict-WebbRubin (BWR) equation, ${ }^{27}$ the densities were estimated and the coefficients of the dilute gases then obtained as functions of temperature. The values for the higher densities and supercritical conditions were obtained by accounting for the density dependences and the critical enhancements. Only a few of the diffusion coefficients 


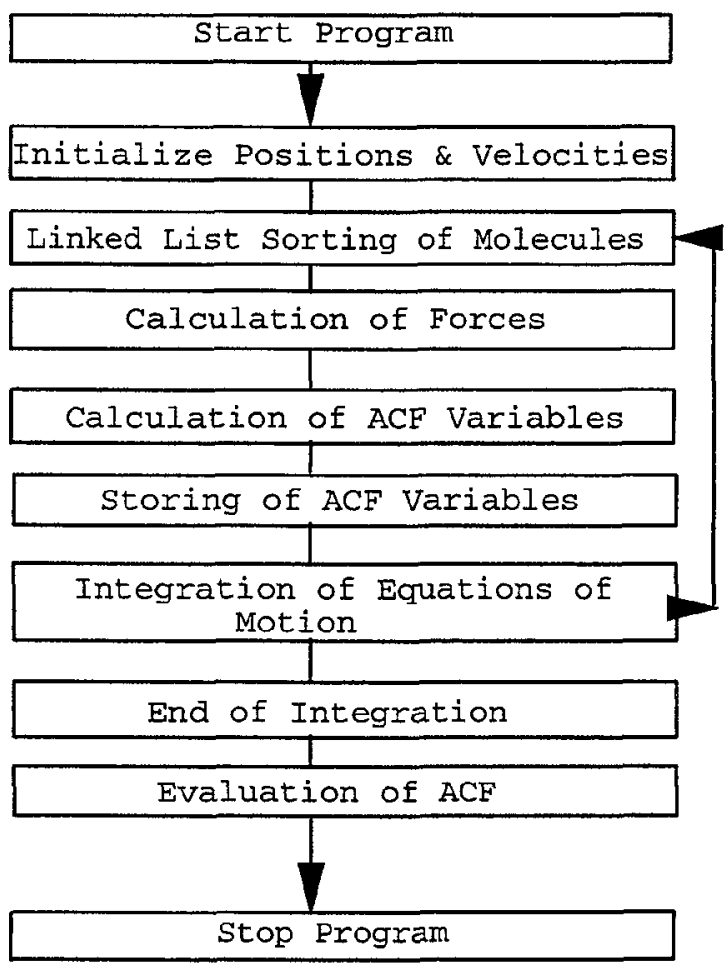

Figure 4: Schematic of MD Simulation Program

computed by the MD simulations are compared to experimental values. ${ }^{28}$ The NIST software does not compute diffusion coefficients. It can be observed that the MD results compare well with the other values, though there are slight discrepancies for some conditions. The discrepancies may be either due to insufficient number of simulation molecules, or due to the oscillatory behavior of the ACF which often results in systematic error in the $\mathrm{MD}$ values.

Table 2: Relevant Parameters of Simulated Substances

\begin{tabular}{||c|c|c|c|c|c||}
\hline Element & $P_{c}$ & $T_{c}$ & $\sigma$ & $\epsilon / k_{B}$ & $\mathrm{~d}$ \\
& $\mathrm{MPa}$ & $\mathrm{K}$ & $\AA$ & $\mathrm{K}$ & $\AA$ \\
\hline Argon & 4.87 & 150.8 & 3.405 & 119.8 & - \\
Nitrogen & 3.39 & 126.2 & 3.293 & 36.5 & 1.094 \\
Oxygen & 5.04 & 154.6 & 2.95 & 5.8 & 1.239 \\
\hline
\end{tabular}

Figure 5 shows plots of diffusion coefficients for oxygen computed via MD. It can be observed that the coefficient decreases with temperature at $3 \mathrm{MPa}$, but increases at the supercritical pressures. Figures 6 and 7 show plots of the shear viscosity, and thermal conductivity as functions of temperature and pressure for oxygen. The MD results compare well with the NIST values especially at the higher temperatures. The MD results do not match the NIST values very well at $160 \mathrm{~K}$ for the $10 \mathrm{MPa}$ and $15 \mathrm{MPa}$ cases. This may be due to the use of insufficient sample size in the simulation as the critical temperature is approached. The maximum difference between the two sets of values was $10.8 \%$ for shear viscosity and $15.1 \%$ for thermal conductivity. Both the shear viscosity and the thermal conductivity can be observed to increase monotonically with temperature at $3 \mathrm{MPa}$. At higher temperatures both quantities tend to be fairly independent of pressure.

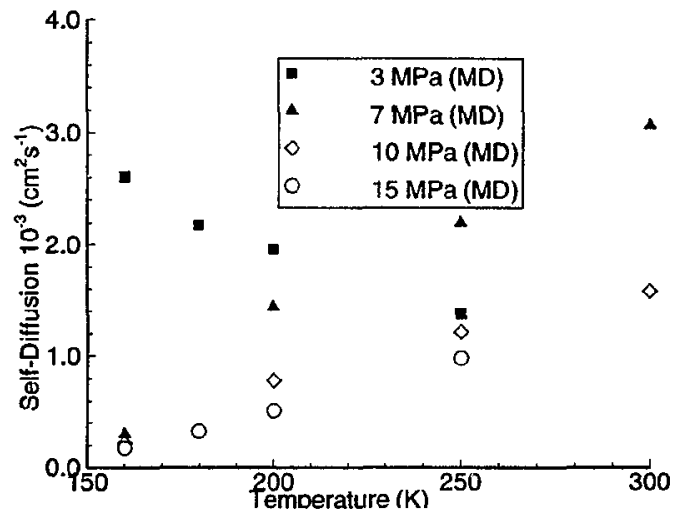

Figure 5: Self-Diffusion Values from MD for oxygen

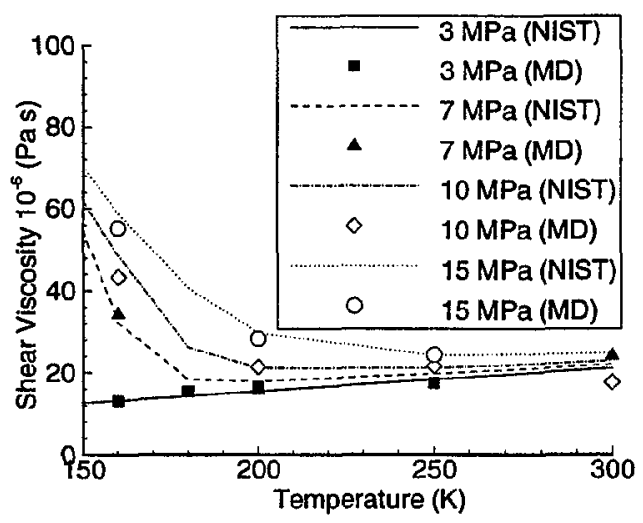

Figure 6: Shear Viscosity Values from MD and NIST for Oxygen

\section{Conclusions}

The transport properties of fluids are being computed via molecular dynamics (MD) simulations using the Green-Kubo formulae. The MD program uses the effective Lennard-Jones potential, with system sizes of 256 molecules and simulations of 500,000 timesteps. Simulations have been carried out on pure argon, nitrogen 
Table 3: Comparison of Various Transport Coefficients Values for Argon.

\begin{tabular}{|c|c|c|c|c|c|c|c|}
\hline $\mathrm{MPa}$ & $T$ & $\begin{array}{c}D \times 10^{5} \\
(\exp ) \\
\mathrm{cm}^{2} s^{-1}\end{array}$ & $\begin{array}{c}D \times 10^{5} \\
(\mathrm{md}) \\
\mathrm{cm}^{2} s^{-1}\end{array}$ & $\begin{array}{c}\mu \times 10^{4} \\
\text { (NIST) } \\
P a s\end{array}$ & $\begin{array}{l}\mu \times 10^{4} \\
(\mathrm{md}) \\
P a s\end{array}$ & $\begin{array}{c}\lambda \times 10^{4} \\
(\mathrm{NIST}) \\
J m^{-1} s^{-1} K^{-1}\end{array}$ & $\begin{array}{c}\lambda \times 10^{4} \\
(\mathrm{md}) \\
J m^{-1} s^{-1} K^{-1}\end{array}$ \\
\hline 0.09 & 86.4 & 1.60 & 1.69 & 2.83 & 2.91 & 132.7 & 152.8 \\
\hline 3.0 & 140 & N/A & 120.9 & 0.137 & 0.145 & 10.8 & 11.1 \\
\hline 3.0 & 300 & $\mathrm{~N} / \mathrm{A}$ & 695.9 & 0.235 & 0.244 & 18.8 & 19.1 \\
\hline 6.0 & 160 & $\mathrm{~N} / \mathrm{A}$ & 35.9 & 0.213 & 0.236 & 27.3 & 31.9 \\
\hline 7.5 & 200 & $\mathrm{~N} / \mathrm{A}$ & 121.3 & 0.202 & 0.213 & 17.0 & 18.2 \\
\hline
\end{tabular}

Table 4: Comparison of Various Transport Coefficients Values for Nitrogen.

\begin{tabular}{|c|c|c|c|c|c|c|c|}
\hline $\mathrm{MPa}$ & $\mathrm{K}$ & $\begin{array}{l}D \times 10^{5} \\
(\mathrm{exp}) \\
\mathrm{cm}^{2} \mathrm{~s}^{-1}\end{array}$ & $\begin{array}{l}D \times 10^{5} \\
(\mathrm{md}) \\
\mathrm{cm}^{2} \mathrm{~s}^{-1}\end{array}$ & $\begin{array}{c}\mu \times 10^{4} \\
\text { (NIST) } \\
P a s\end{array}$ & $\begin{array}{c}\mu \times 10^{4} \\
(\mathrm{md}) \\
P a s\end{array}$ & $\begin{array}{c}\lambda \times 10^{4} \\
(\mathrm{NIST}) \\
J_{m^{-1} s^{-1} K^{-1}}\end{array}$ & $\begin{array}{c}\lambda \times 10^{4} \\
(\mathrm{md}) \\
J m^{-1} s^{-1} K^{-1}\end{array}$ \\
\hline 2.5 & 100 & $\mathrm{~N} / \mathrm{A}$ & 7.0 & 0.765 & 0.747 & 100 & 101 \\
\hline 4.0 & $\overline{120}$ & 18.4 & 21.1 & 0.426 & 0.485 & 62.1 & 55.2 \\
\hline
\end{tabular}

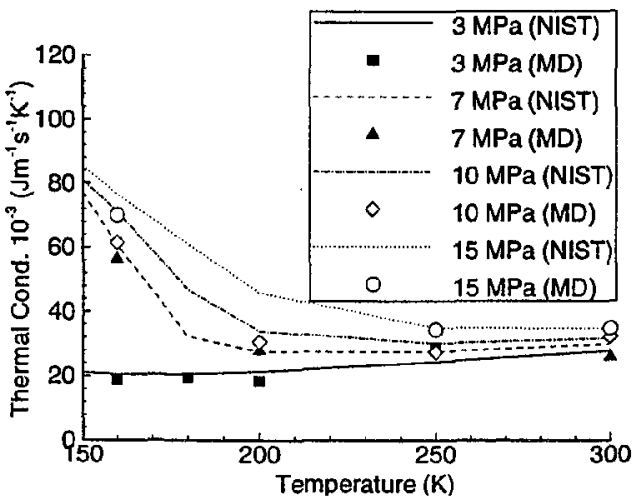

Figure 7: Thermal Conductivity Values from $\mathrm{MD}$ and NIST for Oxygen

and oxygen at various supercritical conditions. The selfdiffusion coefficients obtained from this study compare very well with experimental data, while the shear viscosity and thermal conductivity coefficients also compare well with NIST values. The maximum difference between the two set of values are $10.8 \%$ for shear viscosity and $15.1 \%$ for thermal conductivity.

Future studies will include the computation of the transport properties for liquid mixtures, and the properties at the surface of evaporating droplets in supercritical environments. ${ }^{17,29}$ Detailed study of the error estimates of the simulation will made. In addition, current equa- tion of state models will be compared to MD simulations. A successful implementation of the code should have a great impact on the understanding of the processes occurring in current and future aerospace propulsion systems.

\section{Acknowledgments}

This work was supported by AFOSR Grant F49620-941-0133 and NASA Grants NAGW-1356 Supplement 10, and NGT-10034. The authors would like to thank Dr. Jeff Little and Ms. Teresa Kaltz for their contributions.

\section{References}

[1] Long, L. N., Micci, M. M., Kaltz, T. L., Little, J. K., and Wong, B. C. Submicron droplet modeling using molecular dynamics. AIAA Paper 95-0412, 1995.

[2] Yang, V., Lin, N., and Shuen, J. S. Vaporization of liquid oxygen droplets in supercritical hydrogen environments. Comb. Science and Tech., 97, pp. 247$270,1994$.

[3] Matlosz, R. L., Leipziger, S., and Tosda, T. P. Investigation of liquid drop evaporation in high temperature and high pressure environment. Intl. J. of Heat and Mass Transfer, 15, pp. 831-852, 1972. 
[4] Rosner, D. E., and Chang, W. S. Transient evaporation and combustion of a fuel droplet near its critical temperature. Comb. Science and Tech., 7, pp. 145-158, 1973.

[5] Kadata, T., and Hiroyasu, H. A model for multicomponent droplet vaporization at high ambient pressures. SAE Paper No. 85-0264, 1976.

[6] Jin, J. D., and Borman, G. L. A model for multicomponent droplet vaporization at high ambient pressures. SAE Paper No. 850264, 1985.

[7] Curtis, E. W., and Farrel, P. V. Droplet vaporization in supercritical microgravity environment. IAF Paper 87-384, 36th Congress of the Intl. Astro. Federation, 1987.

[8] Hsieh, K. C., Shuen, J. S., and Yang, V. Droplet vaporization in high-pressure environments, $\mathrm{i}$ : Near critical conditions. Comb. Science and Tech., 76, pp. 111-132, 1991.

[9] Hoheisel, C., and Vogelsang, R. Thermal transport coefficients for one-component and two-component liquids from time correlation functions computed by molecular dynamics. Comp. Phys. Rep., 18, pp. 1$69,1988$.

[10] Hoheisel, C. Transport properties of molecular liquids. Physics Reports, 245, pp. 111-157, 1994.

[11] Chapman, S., and Cowling, T. G. The Mathematical Theory of Non-Uniform Gases. Cambridge University Press, 1970.

[12] Allen, M. P., and Tildesley, D. J. Computer Simulation of Liquids. Clarendon Press, 1983.

[13] Rapaport, D. C. The Art of Molecular Dynamics Simulation. Cambridge University Press, 1995.

[14] Hirschfelder, J. O., Curtiss, C. F., and Bird, R. B. Molecular Theory of Gases and Liquids. John Wiley and Sons, 1954.

[15] Garrison, B. J. Molecular dynamics simulation of surface chemical reaction. Chem. Soc. Review, 21, pp. 155-162, 1992.

[16] Luo, H., and Hoheisel, C. Collective transport in a molecular molecular liquid with quadrupole interaction. Phys. Rev. A, 43, pp. 1819-1825, 1991.

[17] Kaltz, T. L. to be published. Ph.D. thesis, The Pennsylvania State University, 1997.

[18] Hoheisel, C. Theoretical Treatment of Liquids and Liquid Mixtures. Elsevier, 1993.
[19] Haile, J. M. Molecular Dynamics Simulation: Elementary Methods. John Wiley, 1992.

[20] Erpenbeck, J. J. Shear viscosity of the lennardjones fluid near the triple point: Green-kubo results. Phys. Rev. A, 38, pp. 6255-6266, 1988.

[21] Cummings, P. T. Molecular Simulation of NearCritical and Supercritical Fluids. Kluwer Academic Publishers, 1994.

[22] Little, J. K. Simulation of Droplet Evaporation in Supercritical Environments Using Parallel Molecular Dynamics. Ph.D. thesis, The Pennsylvania State University, May 1996.

[23] Plimpton, S. Fast parallel algorithms for shortrange molecular dynamics. J. Comp. Phys., 117, pp. 1-19, 1995.

[24] National Institute of Standards and Technology, Gaithersburg, MD. NIST Thermophysical Properties of Pure Fluids Database: Version 3.0, 1996.

[25] Hanley, H. J. M., McCarty, R. D., and Haynes, W. M. The viscosity and thermal conductivity coefficients for dense gaseous and liquid argon, krypton, xenon, and oxygen. J. Phys. Chem. Ref. Data, 3, pp. 979-1018, 1974.

[26] Younglove, B. A., and Hanley, H. J. M. The viscosity and thermal conductivity coefficients of gaseous and liquid argon. J. Phys. Chem. Ref. Data, 15, pp. 1323-1337, 1986.

[27] Jacobsen, R. T., and Stewart, R. B. J. Thermodynamic properties of nitrogen including liquid and vapor phases from $63 \mathrm{k}$ to $2000 \mathrm{k}$ with pressure to 10,000 bar. J. Phys. Chem. Ref. Data, 2, pp. 757$922,1973$.

[28] Cheung, P. S. Y., and Powles, J. G. The properties of liquid nitrogen. part iv. a computer simulation. Molecular Physics, 20, pp. 921-949, 1975.

[29] Nwobi, O. C. to be published. Ph.D. thesis, The Pennsylvania State University, 1997. 